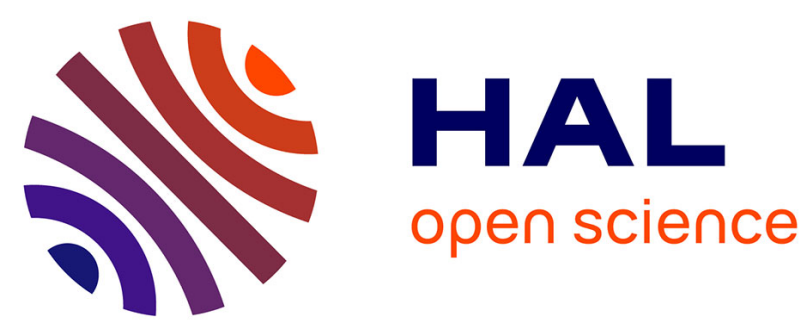

\title{
Shock generation comparison with planar and hemispherical targets in shock ignition relevant experiment
}

S. D. Baton, E. Le Bel, S. Brygoo, X. Ribeyre, C. Rousseaux, J. Breil, M. Koenig, D. Batani, D. Raffestin

\section{To cite this version:}

S. D. Baton, E. Le Bel, S. Brygoo, X. Ribeyre, C. Rousseaux, et al.. Shock generation comparison with planar and hemispherical targets in shock ignition relevant experiment. Physics of Plasmas, 2017, 24 (9), pp.092708. 10.1063/1.4989525 . hal-01828176

\section{HAL Id: hal-01828176 https://hal.science/hal-01828176}

Submitted on 3 Jul 2018

HAL is a multi-disciplinary open access archive for the deposit and dissemination of scientific research documents, whether they are published or not. The documents may come from teaching and research institutions in France or abroad, or from public or private research centers.
L'archive ouverte pluridisciplinaire HAL, est destinée au dépôt et à la diffusion de documents scientifiques de niveau recherche, publiés ou non, émanant des établissements d'enseignement et de recherche français ou étrangers, des laboratoires publics ou privés. 
Shock generation comparison with planar and hemispherical targets in shock ignition relevant experiment

\author{
S.D. Baton ${ }^{1,2}$, E. Le Bel ${ }^{3}$, S. Brygoo ${ }^{4}$, X. Ribeyre ${ }^{3}$, C. Rousseaux ${ }^{4}$, J. Breil ${ }^{3}$, M. Koenig ${ }^{1,2}$, \\ D. Batani ${ }^{3}$, D. Raffestin ${ }^{5}$ \\ ${ }^{1}$ LULI - CNRS, Ecole Polytechnique, CEA, Université Paris-Saclay, F-91128 Palaiseau cedex, France \\ ${ }^{2}$ Sorbonne Universités, UPMC Univ. Paris 06, CNRS, LULI, place Jussieu, 75252 Paris cedex 05, France \\ ${ }^{3}$ Université de Bordeaux-CNRS-CEA, Centre Lasers Intenses et Applications (CELIA), Talence, F-33405, France \\ ${ }^{4} C E A, D A M, D I F, F-91297$ Arpajon, France \\ ${ }^{5}$ CEA, DAM, CESTA, F-33114 Le Barp, France
}

We performed an experiment on the "Ligne d'Intégration Laser" facility to produce strong shocks with plasma conditions relevant for the Shock Ignition approach to Inertial Confinement Fusion. Two kinds of target have been used: planar and hemispherical. We observe an increase of the shock velocity in hemispherical geometry, which entails a fairly planar shock despite the Gaussian focal spot. Numerical results reproduce in the successful way the shock dynamics in the two cases, indicating, for laser intensities around $1.5 \times 10^{15} \mathrm{~W} / \mathrm{cm}^{2}$ at $3 \omega$, an ablation pressure of $(90 \pm 20)$ Mbar and (120 \pm 20$)$ Mbar in planar and hemispherical geometry respectively.

\title{
I. INTRODUCTION
}

Inertial Confinement Fusion (ICF), the classical process leading to deuterium-tritium (DT) ignition, is the conversion of the kinetic energy of the imploding shell into the internal energy of a central hot spot $[1,2]$. An alternative pathway for achieving central ignition, the shock ignition (SI), based on the separation of the compression and the ignition phases, has been considered [3,4]. This scheme is likely to provide higher gains than the conventional approach. Preliminary experiments performed on the OMEGA laser facility have been quite encouraging [5,6,7] and a proof-of-principle experiment on the National Ignition Facility has been proposed [8]. The SI option is also under investigation for the Laser MegaJoule [9], and has been studied in the context of the European directdrive HiPER project $[10,11,12]$.

In the SI scheme, a strong converging shock is launched in the target by a high-intensity $\left(\leq 10^{16}\right.$ $\mathrm{W} / \mathrm{cm}^{2}$ ) laser spike at the end of the compression phase and prior to the final stagnation of fuel. The collision between the converging shock and the one, originating from the reflection at target center of the first shock produced by the compression beams, creates the conditions for ignition. This concept has been studied theoretically [13] and experimentally (see [14] for a recent review). Experiments in planar geometry have addressed the capability of creating a strong shock in presence of a large plasma corona, the impact of parametric instabilities in laser-plasma interaction and of the hot electrons they produce $[15,16,17]$. In spherical geometry $[5,18,19,20]$ it was demonstrated experimentally that a properly timed final shock significantly enhances the neutron yield [5]. Recent encouraging results $[19,20]$ demonstrated a crucial issue for the credibility of SI, i.e. the capability of generating very high pressures ( $\sim 300 \mathrm{Mbar}$ at the ablation front) for laser intensities in the range of $10^{15}$ to $10^{16} \mathrm{~W} / \mathrm{cm}^{2}$.

Shock ignition is a two-step process. First, a uniform illumination compresses the target at low 
implosion velocity and low isentropic assembly. Second, a high-intensity laser spike launches a strong converging shock to ignite the central hot spot. It is worth noticing that when the strong shock is launched, the critical radius of the target is reduced; hence the focal spot becomes not optimized for the igniting beams. The consequence is a bad overlapping of beams and the pointing on the target radius becomes more critical, which should lead to a decrease of the absorption and consequently of the shock strength.

Nevertheless, even if the compression phase requires a uniform spherical irradiation, the simulations show that the irradiation is less restrictive for the ignition spike as observed from a recent numerical work [11]. However, these simulations do not take into account the hot-electron production or magnetic field generation during the high intensity spike, which can modify the shock uniformity during the compression phase. In this configuration, hydrodynamic simulations show that two planar shocks propagate inside the spherical hot spot, collide and bounce to finally ignite the capsule. The production of a quasi-planar shock has also been experimentally observed in another context, i.e. with shock waves driven by ion beam inside spherical mass targets [21].

In this paper, we present an experiment performed on the "Ligne d'Intégration Laser" (LIL) at CEA/CESTA, in order to study the influence of the target shape, which entails a specific ablation front curvature, for producing a strong planar shock by a laser spike pulse in presence of a large plasma corona with parameters relevant for SI. An original configuration is investigated where a planar shock could be produced by using an "usual" Gaussian shaped laser focal spot impinging on a hemispherical target.

\section{EXPERIMENTAL SETUP}

In the experiment, the four laser beams of the LIL quad were focused on two different kinds of target: first, on planar targets to validate the diagnostics and the simulations in a classical case; second, on hemispherical targets in order to observe the behavior of the spike shock in this specific geometry. VISAR (Velocity Interferometer System for Any Reflector) and SOP (Streaked Optical Pyrometry) diagnostics allowed following the shock wave propagation in time. In order to infer all shock characteristics, experimental results were compared to detailed numerical simulations performed with the CHIC code [22].

The LIL facility delivered $10 \mathrm{~kJ}$ total laser energy on target at $351 \mathrm{~nm}(3 \omega)$ wavelength [23]. In addition to continuous phase plate, two-phase modulators incorporated in the front-end laser temporally smoothed the beams. Their goals are to mitigate plasma instabilities (and hydrodynamic instabilities in direct drive experiments), as well as to improve the propagation of the beam through large plasmas by reducing laser filamentation [24].

We used a temporally shaped laser pulse (Fig. 1b): a 2 ns square pulse is assimilated to the final ignition spike at peak power $\sim 4 \mathrm{TW}$, yielding a mean intensity on target of $1.5 \times 10^{15} \mathrm{~W} / \mathrm{cm}^{2}$; it is 
preceded by a $1 \mathrm{~ns}$ pedestal at lower intensity $(\sim 1 \mathrm{TW})$.

The 2ns square pulse has been chosen in order to sufficiently sustain for a long time, in planar geometry, the shocks and in proportion to the $\mathrm{CH}$ ablator thickness (>200 microns). The quad was focused by a $\mathrm{f} / 8$ focusing system with continuous phase plates designed to produce a fairly small focal spot in order to reach the highest intensity as possible for producing a strong shock.

The target, either plane or hemispherical, consisted of three layers (Fig. 1a): (i) a $270 \mu \mathrm{m}$ plastic $(\mathrm{CH})$ ablator to absorb the incident laser light and to reduce the X-ray production; (ii) a $30 \mu \mathrm{m} \mathrm{Mo}$ layer to block X-rays from the coronal plasma; (iii) the rear part of the target was a $250 \mu \mathrm{m} \alpha$-quartz window for VISAR and SOP diagnostics. The first $\mathrm{CH}$ layer was either planar (270 $\mu \mathrm{m}$ thickness) or an hemisphere of $500 \mu \mathrm{m}$ diameter attached onto $20 \mu \mathrm{m} \mathrm{CH}$ planar layer. Hence, the $\mathrm{CH}$ thickness at the top of hemispherical target was the same than the planar one. The diameter of the hemispherical part of the target has been chosen to match the LIL focal spot, measured to be Gaussian with a radius of $180 \mu \mathrm{m}($ at $1 / \mathrm{e})$. The laser beam is perpendicular to the target and is focused on the center of the hemisphere (cf. Fig. 1c).

A set of two VISARs [25], one at $\lambda=1064 \mathrm{~nm}$ and one at $\lambda=532 \mathrm{~nm}$, and a SOP at $650 \mathrm{~nm}$ were used to record as a function of time the shock velocity and the self-emission of the target rear side. The self-emission was also recorded with a GOI (Gated Optical Imager) at $650 \mathrm{~nm}$ giving an instantaneous 2D image with a 400 ps gate width. Laser plasma instabilities have also been measured: the light backscattered through the $\mathrm{f} / 8$ focusing cone was collected by the FABS (Full Aperture Backscattered) diagnostic. The $3 \omega$ light, backscattered around the focusing cone, was gathered by NBI (Near-Backscattered Imaging) diagnostics, providing the amount of stimulated Brillouin scattering (SBS) energy scattered between $\mathrm{f} / 4$ and $\mathrm{f} / 2$ apertures [26].

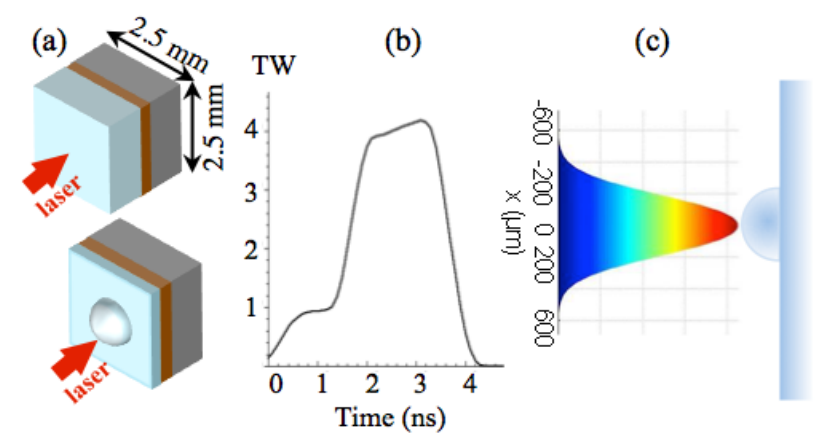

FIG. 1 (color online). (a) schematic drawing of the planar (up) and hemispherical (bottom) targets; (b) laser temporal pulse shape; (c) schematic of the laser focal spot on the hemisphere

\section{EXPERIMENTAL RESULTS}

The energy fraction of the total backscattered light (SRS, stimulated Raman scattering, and SBS) through the $\mathrm{f} / 8$ focusing cone has been measured around $5 \%$ with respect to the total incident laser 
energy and less than $8 \%$ with respect to the laser energy encircled in a $500-\mu \mathrm{m}$ diameter (which is the diameter of the hemispherical target). The energy fraction of SBS collected by the NBI diagnostics was always low, less or equal to $\approx 1.5 \%$ (we do not have this measurement for Raman). About $2 / 3$ of the reflected energy is due to SBS and 1/3 to SRS. As an example, Fig. 2 shows SBS and SRS powers measured in $\mathrm{f} / 8$ for the two shots discussed below, Fig. $2 \mathrm{a}$ for the planar target and Fig. $2 \mathrm{~b}$ for the hemispherical one. Backscattered SBS predominantly develops during the rising part of the laser spike, where the ion acoustic wave damping is expected to be low (because of low ion temperature) as well as the electron temperature for which the SBS inhomogeneous threshold is proportional. The peak SBS reflectivity reaches $6.5 \%$ for the planar target (the time-averaged SBS reflectivity is $2.5 \%$ on this shot). On the contrary, SRS develops more smoothly during the high intensity part of the laser pulse, but the peak SRS reflectivity is lower, $3 \%$ on this shot (and $1.2 \%$ for the time-averaged value). Generally, the SBS and SRS measurements are observed to be approximately twice weaker for hemispherical targets compared to the planar targets.
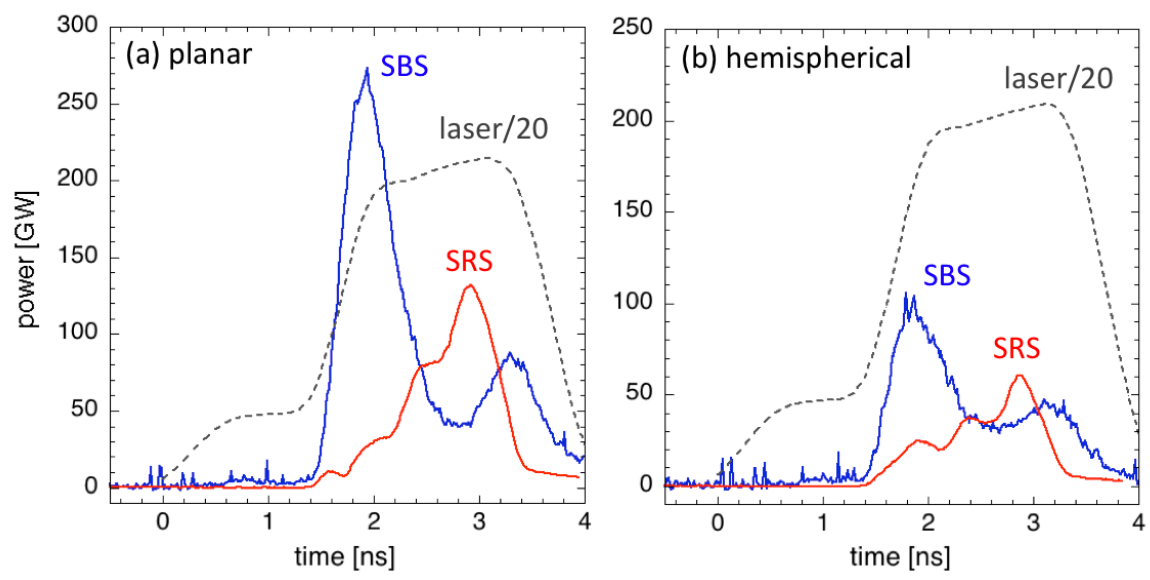

FIG. 2 (color online): Time-resolved powers of SBS (in blue) and SRS (in red) measured in f/8 for a planar target (a) and a hemispherical target (b). The incident laser power (divided by 20) is also shown (dashed gray line).

These relatively modest reflectivity values for laser intensities as high as $1.5 \times 10^{15} \mathrm{~W} / \mathrm{cm}^{2}$ have already been observed in previous SI experiments $[15,17,18]$. Low reflectivity is a consequence of the combination between the interaction with laser beams using improved temporal smoothing technique and inhomogeneous plasmas. In the experiment, the inhomogeneity affects both the electron density profile (detuning SRS and SBS) and the expansion velocity of the plasma into the vacuum, altering the resonance conditions of SBS. This is illustrated in Fig. 3 showing the SRS and SBS thresholds [27] as calculated for plasma conditions given by our 2D hydrodynamic CHIC code [22]. It is shown that the SRS threshold occurs around $2-4 \times 10^{15} \mathrm{~W} / \mathrm{cm}^{2}$, so that the intensity threshold might be marginally reached in our experiment. For SBS, Fig. 3 shows that the effect of the 
velocity detuning is more significant than the density profile one. However, the SBS threshold is below the SRS one, around 2 to $5 \times 10^{14} \mathrm{~W} / \mathrm{cm}^{2}$, that qualitatively explain the SBS prevalence over SRS, in agreement with the data. Comparing now these thresholds in plasma profiles expected from targets designed for the direct-drive scheme for LMJ [28], we note that the electron density scalelength is typically one-third smaller in our experiments, thus the SRS threshold is expected to be one third lower as well for LMJ. On the contrary, both the electron temperature $T_{e}$ and the expansion velocity scale-length $\mathrm{L}_{\mathrm{V}}=[1 / \mathrm{V} \Delta \mathrm{V} / \Delta \mathrm{z}]^{-1}$ increase by a factor of two in the LMJ design, as calculated by the CHIC code. However, as the SBS threshold is proportional to the ratio $\mathrm{T}_{\mathrm{e}} / \mathrm{L}_{\mathrm{V}}$, the threshold for LMJ is expected to be comparable to the current experiment one.

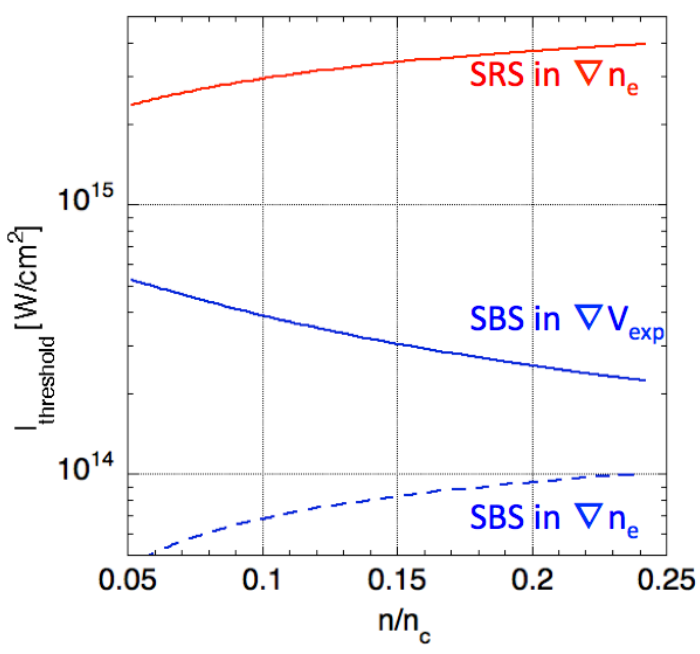

FIG. 3 (color online): convective intensity thresholds as a function of the electron density in the corona, expected in our experiments, for SRS (red) and SBS (blue) (from [27]). For SBS, the detuning caused by the plasma expansion is more severe than by the electron density profile one.

In Fig. 4 are shown the shock-related data for a planar target. They are plotted as a function of time, $t=0 \mathrm{~ns}$ corresponding to the onset of the laser pulse. Fig. 4(a) shows the 2D self-emission image obtained from the GOI taken at $\mathrm{t} \sim 8 \mathrm{~ns}$. The signal is circular with a diameter matching the SOP profile at the same time. The bright signal in SOP (Fig. 4b) seen at $t \sim 1.6$ ns coincides with the laser spike hitting the target and generating X-ray radiation from the ablation plasma. X-ray, as well as hot electrons generated by SRS or Two-Plasma Decay instabilities, propagate through the target and can ionize the material ahead of the shock wave changing of its refractive index. This produces a perturbation in VISAR image (Fig. 4c) with a slight drop of the signal at $t \sim 1.6 \mathrm{~ns}$ and a complete blanking where the intensity is the strongest. This phenomenon was previously observed in other experiments using VISAR optical probe beam [29, 30]. At $\mathrm{t} \sim 4.3 \mathrm{~ns}$, the shock breakout into the quartz is accompanied by light emission recorded by the SOP diagnostic and a strong fringe shift is observed in the VISAR image. Due to the curvature of the shock, only one fringe, superimposed to weaker ghost fringes, is visible: the non-planar shock is strongest and fastest in the center, 
corresponding to the maximum laser intensity, and decreases strongly on the edges. The strong curvature of the shock front reflects most of the probe light outside of the collecting aperture of the diagnostic. At $\sim 10.8 \mathrm{~ns}$, the shock breaks out into vacuum, as evidenced by the loss of signal in both images. Calibration shots were performed prior to the experiment in order to calibrate the counts from SOP as a function of the velocity given by the VISAR. Consequently, when the fringes are absent due to the shock curvature, we can extract velocity information directly from the SOP. As the thermal emission is isotropic, SOP signal is indeed less sensitive to the shock curvature than the VISAR signal.

\section{2D HYDRODYNAMIC SIMULATIONS AND DISCUSSION}

The numerical simulations presented in this paper have been performed with $\mathrm{CHIC}$, a 2D radiative ALE (Arbitrary Lagrangian Eulerian) hydrodynamic code [22]. For the interface reconstruction, we used a multi-material treatment of the cells based on the Moment of Fluid method $[31,32]$, in order to correctly track the strong deformations of hemispheric targets during the experiment. The Fig. 4(d) compares velocities obtained from VISAR, SOP (with error bars $~ 5 \%$ giving an uncertainty of $\pm 1 \mathrm{~km} / \mathrm{s}$ ), and the data from the code.
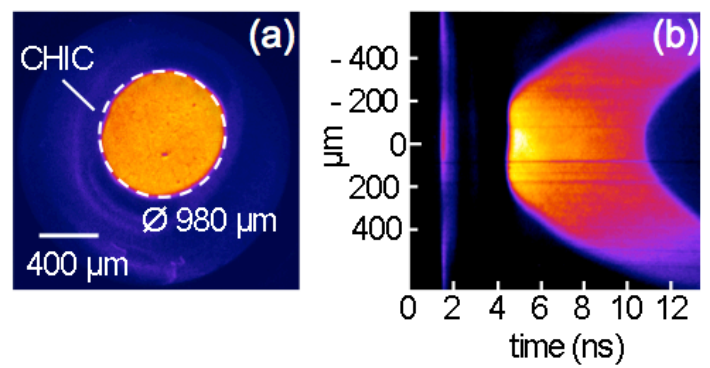
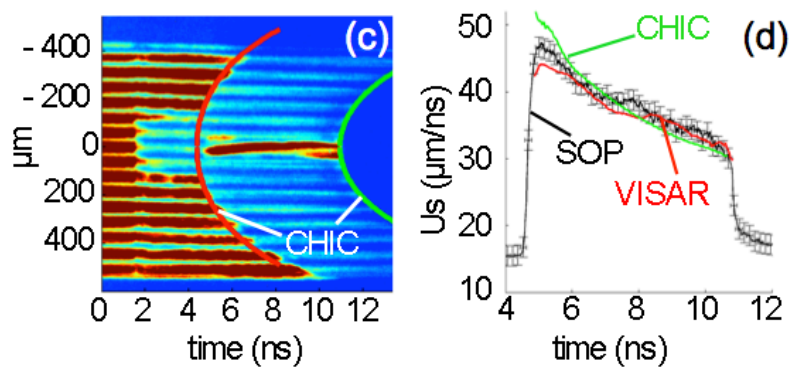

FIG. 4 (color online). Experimental shock propagation data obtained with (a) GOI, (b) SOP and (c) VISAR with a planar target. (d) shows extracted shock velocities from VISAR, SOP and calculated from CHIC.

Given the error bars, numerical simulations are in fairly good agreement with experimental results. At early times, the calculated velocity seems slightly faster than what was observed in the experiment. This slight disagreement could be explained by the blanking of quartz just after shock breakout, making data analysis more difficult. The shock breakout time into vacuum at the rear side of the quartz window ( $\mathrm{t} \approx 11 \mathrm{~ns}$ ), the spatial shape of shock breakout on the VISAR image (plain lines in Fig. 4c) as well as the shape of the self-emission on the GOI image (dashed line in Fig. 4a) are properly reproduced by the simulations. This means that, using the actual intensity distribution in the focal spot, the code correctly predicts the shock strength over the focal spot area as well as its $2 \mathrm{D}$ propagation. The experimental/numerical agreement in the shock dynamics and the velocity for 
planar target is better than $3 \%$, indicating a good numerical treatment of laser-target interaction and ablation physics. This gives a good confidence in the capability of reproducing shock propagation in a more complex geometry.

Figure 5(a) shows the VISAR image for a hemispherical target. The four specific events indicated on the figure relate to: the shock breakout into the quartz (A) and into vacuum (D) for the edge of the laser focal spot, outside the hemisphere; the shock breakout into the quartz (B) and into vacuum (C) for the focal spot center. Figure 5(b) displays a 2D image of the self-emission taken at $\mathrm{t} \sim 8 \mathrm{~ns}$. The brightest round signal corresponds to self-emission in the axis of the hemisphere. It is centered on a weaker signal, attributed to the self-emission from the shock generated by the wings of the focal spot. This also indicates that the laser spot was well centered on the hemisphere. Table 5(c) summarizes the times of these four events, from the experiment and the simulation. Despite the complex geometry and target design, we observe a good agreement (better than 3\%), not only for the absolute times, but also for the shock shape. This is highlighted by the red (green respectively) contour line shown in Fig. 5(a) representing the calculated shock front at shock breakout into quartz (into the vacuum respectively), that properly match the experimental features. Likewise, the dashed line representing the calculated shape of the self-emission fits correctly the GOI image (Fig. 5b)
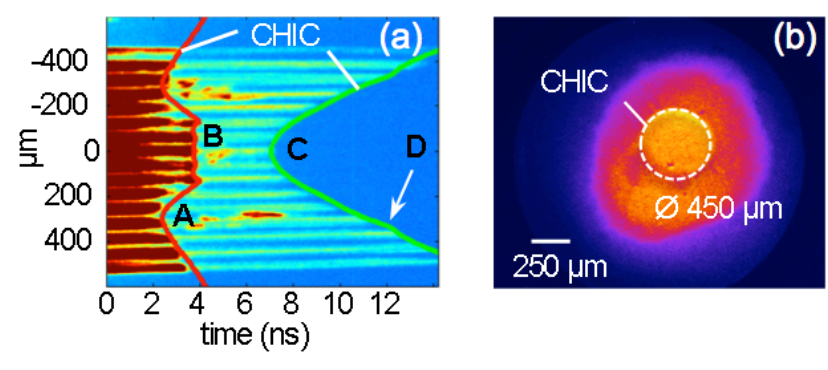

\begin{tabular}{ccc}
\hline \hline & Time /t0 exp. (ns) & Time /t0 code (ns) \\
(c)
\end{tabular}

FIG. 5 (color online). Experimental shock propagation data obtained with (a) VISAR and (b) GOI at $\mathrm{t} \sim 8 \mathrm{~ns}$ for a hemispherical target. Table (c) displays a comparison of data obtained from experiment and 2D hydrodynamic CHIC code for specific events indicated on the VISAR image in (a).

It is also important to notice the shock emerges with a flat front at the centre of the hemisphere after its propagation. This is the result of the balance between the laser intensity and the target geometry. The laser intensity, thus the shock velocity [33], is maximum at the pole of the $\mathrm{CH}$ hemisphere while it decreases across the diameter where the laser interacts both at an increased incident angle and with a reduced $\mathrm{CH}$ thickness. The combination of these effects, which compensate themselves, straightens the shock front along its propagation into the hemisphere leading to a flat shock front near the $\mathrm{Mo} / \mathrm{SiO}_{2}$ interface. This is clearly observed on Fig. 6(A) and 6(B), which represent the density maps at the times corresponding to the events cited in Fig. 5(a). These simulations allow following the time evolution of the shock front. Because the wings of the laser 
spot are larger than the hemisphere, an early shock breakout into quartz (A) is observed at $\sim 2.4 \mathrm{~ns}$, before the breakout of the central flat shock (B) at $3.7 \mathrm{~ns}$. The central flat shock propagates faster in the quartz and reaches the vacuum at $\sim 7.1 \mathrm{~ns}$ (C). Finally, the annular slow shock front and the fast central shock are catching up at the rear face of the quartz at $12.2 \mathrm{~ns}$ (D) leading to a change of the shock front curvature faintly observed on VISAR image (Fig. 5(a) point D).

As the shock dynamics is reproduced in details, we can use our numerical simulations to infer the ablation pressure generated by the laser pulse. Just after the spike beam has reached its maximum, we obtain a value of $(90 \pm 20)$ Mbar and $(120 \pm 20)$ Mbar with planar and hemispherical targets respectively. Considering the 1D scaling law of the ablation pressure $\mathrm{P}_{\mathrm{abl}}$ in the regime of classical absorption, $\mathrm{P}_{\mathrm{abl}} \sim 57\left(\eta_{\text {abs }} \mathrm{I}_{\mathrm{L}} / \lambda\right)^{2 / 3}[34,35]$, where $\eta_{\text {abs }}$ is the laser absorption fraction, $\mathrm{I}_{\mathrm{L}}$ denotes the laser intensity in units of $10^{15} \mathrm{~W} / \mathrm{cm}^{2}$ and $\lambda$ is the laser wavelength in $\mu \mathrm{m}$, we obtain, in our experimental conditions and assuming $80 \%$ absorption, an ablation pressure of $130 \mathrm{Mbar}$. This value agrees with the one inferred from numerical simulations in hemispherical geometry where the planar shock outlasts in the planar geometry. In the last case, the energy losses due to lateral conduction play an important role, reducing the actual ablation pressure.
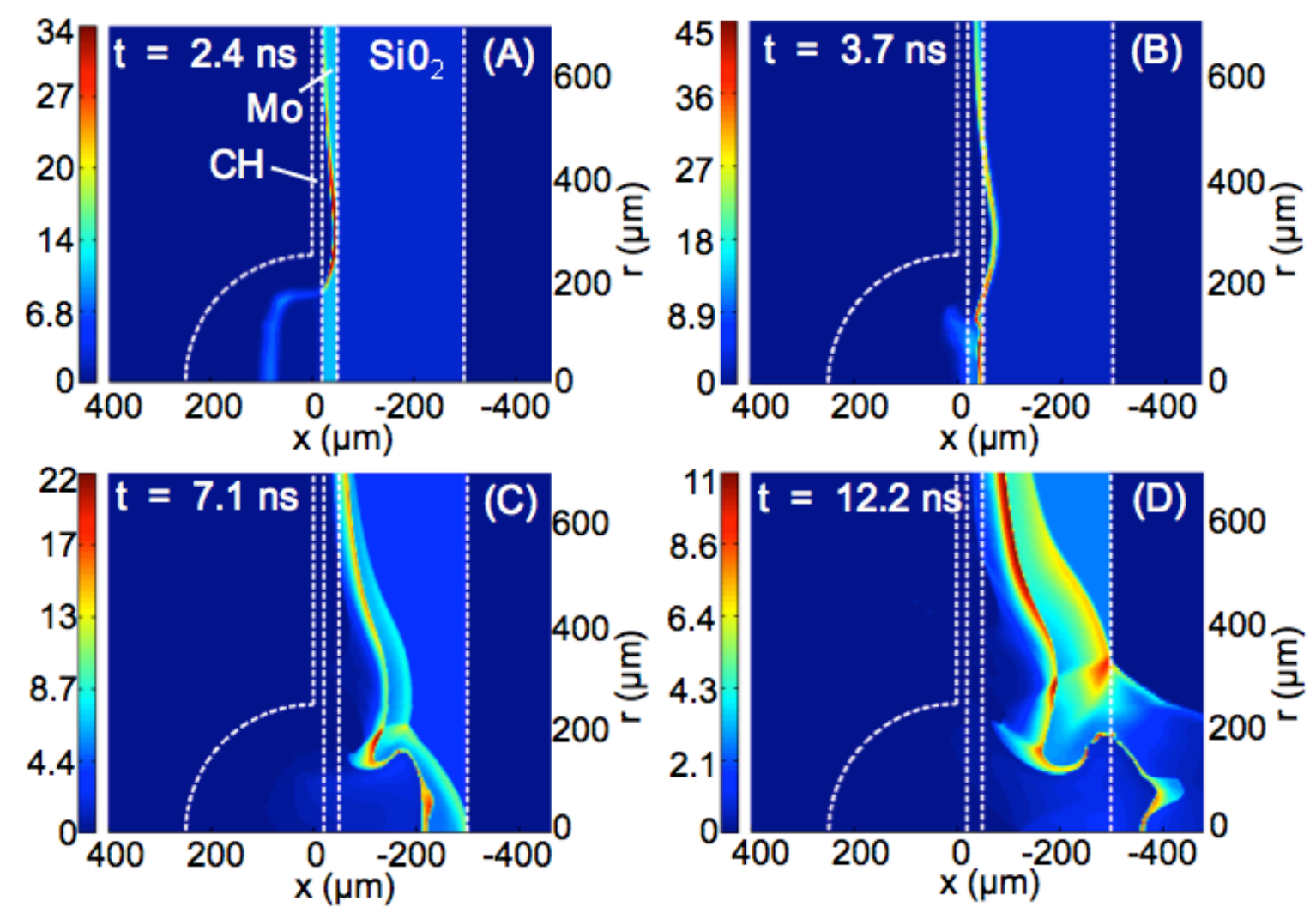

FIG. 6 (color online). Maps of density (in $\mathrm{g} / \mathrm{cc}$ ) at the four times corresponding to the events indicated on the VISAR image displayed in Fig. 5(a). The dashed lines represent the initial interface positions between the three layers $\mathrm{CH} / \mathrm{Mo} / \mathrm{SiO} 2$. The laser comes from the left. 
Our experimental results confirm that we are able to launch very strong shocks in the presence of a large plasma corona in realistic ICF conditions and that hemispherical targets generate higher ablation pressures. This is the consequence of the lateral energy flow, spreading away from the focal spot region, in the case of planar target, thus leading to a non-sustained shock. While, due to the geometry counterbalancing such lateral flow, hemispherical targets provide higher ablation pressures.

\section{CONCLUSION}

In summary, we have investigated the behavior of a shock produced by a laser spike at intensity around $1.5 \times 10^{15} \mathrm{~W} / \mathrm{cm}^{2}$ in the presence of a pre-plasma within conditions relevant to shock ignition, in planar and hemispherical geometries. The backscattered light (SBS and SRS) is relatively low, limited to $\sim 5 \%$ in the focusing cone. $2 \mathrm{D}$ hydro-simulations reproduce the shock dynamics in the two cases, and correctly predict the timing and the shape of the shocks allowing to estimate an ablation pressure of $90 \mathrm{Mbar}$ and $120 \mathrm{Mbar}$ in planar and spherical geometry respectively. The increase of the shock velocity in hemispherical $\mathrm{CH}$ targets is due to reduced lateral conduction and energy losses, which become important in planar target. With hemispherical targets, the interplay between the target geometry and the spatial intensity distribution entails a final planar shock across the diameter, in spite of using a small focal spot generating an initially curved shock. This could be exploited in the polar direct drive geometry (i.e. with spherical capsule) in order to produce two strong flat shocks colliding at target center to possibly reaching the conditions for fuel ignition [11, 36, 37].

\section{ACKNOWLEDGMENTS}

The authors gratefully acknowledge the technical assistance of all LIL teams, and the CEAValduc target laboratory for providing high quality targets. This work was supported by the Institute Laser-Plasma (ILP) and within the framework of the EUROfusion Consortium via the ToIFE project. It has received funding from the Euratom research and training programme 2014-2018 under grant agreement $\mathrm{N}^{\circ}$ 633053. The views and opinions expressed herein do not necessary reflect those of European Commission.

[1] S. Atzeni and J. Meyer-ter-Vehn, The Physics of Inertial Fusion (Clarendon Press, Oxford, 2004). [2] J. D. Lindl, Inertial Confinement Fusion (Springer, New York, 1998).

[3] R. Betti, C.D. Zhou, K.S. Anderson, L.J. Perkins, W. Theobald, and A.A. Solodov, Phys. Rev. Lett. 98, 155001 (2007).

[4] V.A. Shcherbakov, Sov. J. Plasma Phys. 9, 240 (1983).

[5] W. Theobald, R. Betti, C. Stoeckl, K. S. Anderson, J. A. Delettrez, V. Yu. Glebov, V. N. 
Goncharov, F. J. Marshall, D. N. Maywar, R. L. McCrory et al., Phys. Plasmas, 15, 056306 (2008). [6] W. Theobald, K.S. Anderson, R. Betti, R.S. Craxton, J.A. Delettrez, J.A. Frenje, V. Yu Glebov, O.V. Gotchev, J.H. Kelly, C.K. Li et al., Plasma Phys. Control. Fus. 51, 124052 (2009).

[7] P. B. Radha, R. Betti, T. R. Boehly, J. A. Delettrez, D. H. Edgell, V. N. Goncharov, I. V. Igumenshchev, J. P. Knauer, J. A. Marozas, F. J. Marshall et al., IEEE Trans. Plasma Science, 39, 1007 (2011).

[8] L.J. Perkins, R. Betti, K. N. LaFortune, and W.H. Williams, Phys. Rev. Lett. 103, 045004 (2009).

[9] B. Canaud and M. Temporal, New J. Phys. 12, 043037 (2010).

[10] S. Atzeni, Plasma Phys. Controlled Fusion 51, 124029 (2009).

[11] X. Ribeyre, G. Schurtz, M. Lafon, S. Galera, and S. Weber, Plasma Phys. Controlled Fusion 51, 015013 (2009).

[12] D. Batani, M. Koenig, S.D. Baton, F. Perez, L.A. Gizzi, P. Koester, L. Labate, J. Honrubia, L. Antonelli, A. Morace et al., Plasma Phys. Control. Fusion 53124041 (2011).

[13] S. Atzeni , X. Ribeyre, G. Schurtz, A.J. Schmitt, B. Canaud, R. Betti and L.J. Perkins, Nucl. Fusion 54, 054008 (2014).

[14] D. Batani, S. Baton, A. Casner, S. Depierreux, M. Hohenberger, O. Klimo, M. Koenig, C.

Labaune, X. Ribeyre, C. Rousseaux, et al. Nucl. Fusion 54, 054009 (2014).

[15] S.D. Baton, M. Koenig, E. Brambrink, H. P. Schlenvoigt, C. Rousseaux, G. Debras, S. Laffite, P. Loiseau, F. Philippe, X. Ribeyre, and G. Schurtz, Phys. Rev. Lett. 108, 195002 (2012).

[16] M. Hohenberger, W. Theobald, S. X. Hu, K. S. Anderson, R. Betti, T. R. Boehly, A. Casner, D. E. Fratanduono, M. Lafon, D. D. Meyerhofer et al., Phys. Plasmas 21, 022702 (2014).

[17] D. Batani, L. Antonelli, S. Atzeni , J. Badziak, F. Baffigi, T. Chodukowski , F. Consoli , G.

Cristoforetti, R. De Angelis , R. Dudzak et al., Phys. Plasmas, 21, 032710 (2014).

[18] W. Theobald, R. Nora, M. Lafon, A. Casner, X. Ribeyre, K. S. Anderson, R. Betti, J. A.

Delettrez, J. A. Frenje, V. Yu. Glebov et al. Phys. Plasmas 19, 102706 (2012).

[19] W. Theobald, R. Nora, W. Seka, M. Lafon, K. S. Anderson, M. Hohenberger, F. J. Marshall, D.

T. Michel, A. A. Solodov, C. Stoeckl et al., Phys. Plasmas 22, 056310 (2015).

[20] R. Nora, W. Theobald, R. Betti, F. J. Marshall, D. T. Michel, W. Seka, B. Yaakobi, M. Lafon, C. Stoeckl, J. Delettrez, et al., Phys. Rev. Lett. 114, 045001 (2015).

[21] A. Henig, D. Kiefer, M. Geissler, S.G. Rykovanov, R. Ramis, R. Hörlein, J. Osterhoff, Zs.

Major, L. Veisz, S. Karsch et al., Phys. Rev. Lett. 102, 095002 (2009).

[22] P.-H. Maire, R. Abgrall, J. Breil and J. Ovadia, SIAM. J. Sci. Comput. 29, 1781 (2007).

[23] X. Julien, A. Adolf, E. Bar, V. Beau E. Bordenave, T.Chiès, R. Courchinoux, J.-M. Di-Nicola,

C. Féral, P. Gendeau et al., Proc. of SPIE Vol. 7916, 791610 (2011).

[24] S. H. Glenzer, D. H. Froula, L. Divol, M. Dorr, R. L. Berger, S. Dixit, B. A. Hammel, C.

Haynam, J. A. Hittinger, J. P. Holder et al., Nat. Phys. 3, 716 (2007).

[25] P. M. Celliers, D. K. Bradley, G. W. Collins, D. G. Hicks, T. R. Boehly, and W. J. Armstrong,

Rev. Sci. Instrum. 75, 4916 (2004).

[26] C. Rousseaux, G. Huser, P. Loiseau, M. Casanova,1 E. Alozy, B. Villette, R. Wrobel,

O. Henry, and D. Raffestin, Phys. Plasmas 22, 022706 (2015).

[27] W. L. Kruer, The physics of laser plasma interactions, Frontiers in Physics, Addison-Wesley Publishing Company, New York, 1988.

[28] A. Colaïtis, X. Ribeyre, E. Le Bel, G. Duchateau, Ph. Nicolaï, and V. Tikhonchuk, Phys. Plasmas 23, 072703 (2016).

[29] S. Laffite, S. D. Baton, P. Combis, J. Clerouin, M. Koenig, V. Recoules, C. Rousseaux, and L.

Videau, Phys. Plasmas 21, 082705 (2014).

[30] W. Theobald J. E. Miller, T. R. Boehly, E. Vianello, D. D. Meyerhofer, T.C. Sangster, J. Eggert,

Baton et al. 
and P. M. Celliers, Phys. Plasmas 13, 122702 (2006).

[31] J. Breil, S Galera, PH Maire, Comput. and Fluids 46, 161 (2011).

[32] M. Billaud Friess, J. Breil, P.-H. Maire, M. Shashkov, Commun. Comput. Phys. 15, 330 (2014).

[33] M. Koenig, B. Faral, A. Benuzzi, D. Batani, S. Bossi, and J. M. Boudenne, Phys. Rev. E 50, R3314 (1994).

[34] R. Fabbro, C. Max and E. Fabre, Phys. Fluids 28, 1463 (1985).

[35] J. Lindl, Phys. Plasmas 2, 3933 (1995).

[36] S. Skupsky, J. A. Marozas , R. S. Craxton, R. Betti , T. J. B. Collins , J. A. Delettrez, V. N. Goncharov , P. W. McKenty , P. B. Radha, T. R. Boehly et al., Phys. Plasmas 11, 2763 (2011).

[37] M. Hohenberger, B. Radha, J.F. Myatt, S. LePape, J.A. Marozas, F.J. Marshall, D.T. Michel, S.P. Regan, W. Seka, A. Shvydky et al., Phys. Plasmas 22, 056308 (2015). 